\title{
Hábito de pastejo de vacas lactantes Holandês x Zebu em pastagens de Brachiaria brizantha e Brachiaria decumbens
}

\author{
[Grazing behavior of Holstein $\times$ Zebu lactating cows in pastures of \\ Brachiaria brizantha and Brachiaria decumbens]

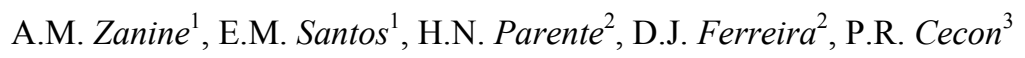 \\ ${ }^{1}$ Aluno de pós-graduação - UFV - Viçosa, MG \\ ${ }^{2}$ Aluna de graduação - UFRRJ - Seropédica, RJ \\ ${ }^{3}$ Departamento de Informática - UFV \\ Av. P.H. Rolfs, $\mathrm{s} / \mathrm{n}$ \\ 36570-000 - Viçosa, MG
}

\begin{abstract}
RESUMO
Avaliou-se o comportamento ingestivo de pastejo de vacas leiteiras em pastagens de Brachiaria brizantha e Brachiaria decumbens, em sistema de lotação contínua com taxa de lotação variável. O delineamento experimental foi o inteiramente ao acaso, com os dois pastos representando os tratamentos, cada um com cinco repetições. O período experimental foi de 30 dias, sendo 20 para adaptação dos animais e 10 para avaliações, em número de três, com duração de $24 \mathrm{~h}$ cada, em intervalos de cinco dias. $\mathrm{O}$ tempo de pastejo da Brachiaria brizantha foi menor, 10,82h, que o tempo de pastejo da Brachiaria decumbens, 12,86h. Comportamento oposto foi observado para o tempo de ruminação, 7,95 e 6,45h para os pastos de Brachiaria brizantha e Brachiaria decumbens, respectivamente. A quantidade de bocados por minuto foi menor na Brachiaria decumbens, 37,30, e maior na Brachiaria brizantha, 42,12. Houve adequação entre os tempos de pastejo, ruminação e taxa de bocados, de modo que o total de bocados e o tempo de ócio não foram diferentes entre os dois pastos.
\end{abstract}

Palavras-chave: vaca de leite, comportamento, bocados, pastejo, ócio

\begin{abstract}
The grazing behavior of lactating cows in pastures of Brachiaria brizantha and Brachiaria decumbens, in system of continuous stocking with variable stocking rate was evaluated. A completely randomized experimental design, with two treatments (pastures) and five replicates was used. The experimental period last 30 days, 20 for adaptation of the animals and 10 for three experimental evaluations, of 24h each, in intervals of five days. The grazing time of cows on the Brachiaria brizantha pasture, 10,82h, was lower than on the Brachiaria decumbens, 12,86h. Inverse behavior was observed for rumination time, 7,95 and 6,45h for cows on the pastures of Brachiaria brizantha and Brachiaria decumbens, respectively. The amount of bite per minute was lower for cows on the Brachiaria decumbens, number 37,30h, than on the Brachiaria brizantha, 42,12h. There was an adequacy among grazing time, rumination time and rate of bites and, as a consequence, no difference between pastures was observed for total of bites and time of idling.
\end{abstract}

Keywords: dairy cow, animal behavior, bite, grazing, idling

Recebido em 5 de março de 2005

Aceito em 30 de novembro de 2006

E-mail: anderson.zanine@ibest.com.br 


\section{INTRODUÇÃO}

As gramíneas do gênero Brachiaria, em especial a Brachiaria decumbens e a Brachiaria brizantha, e outras, em menor escala, ocupam cerca de $85 \%$ das pastagens na região dos cerrados (42,5 milhões de hectares). Conhecidas no Brasil desde a década de 50 , a verdadeira expansão desse gênero ocorreu nas áreas de cerrado nas décadas de 70 e 80 , principalmente nas regiões de clima mais quente (Zimmer et al., 1988).

O ecossistema de pastagens é caracterizado por uma série de inter-relações, dentre elas a interface planta-animal, regida por relações causa-efeito, em que diferentes estruturas do dossel forrageiro determinam padrões distintos de comportamento e desempenho animal (Sarmento, 2003)

O conhecimento dos ciclos diários de pastejo dos animais, assim como o tempo gasto por dia para essa atividade, são fatores de grande relevância em sistemas de produção em pasto. A definição dos horários em que preferencialmente os animais exercem o pastejo é importante para o estabelecimento de estratégias adequadas de manejo. O tempo total gasto para o pastejo é um fator relacionado ao consumo voluntário, com maior ou menor gasto de energia (Ribeiro et al., 1999).

$\mathrm{O}$ animal em pastejo pode selecionar a dieta, desse modo, o pastejo seletivo permite compensar a baixa qualidade da forragem, possibilitando a ingestão de partes mais nutritivas das plantas (Olivo et al., 2004). Entretanto, o comportamento seletivo promove aumento no tempo total de pastejo.

Admitindo-se que o bocado é a unidade básica para obtenção de nutrientes, Carvalho (2000) sintetizou o processo de pastejo em três etapas, não necessariamente excludentes: a) tempo de procura pelo bocado; b) tempo para a ação do bocado e c) tempo para a manipulação do bocado.

Segundo Minson e Wilson (1994), as características químico-bromatológicas, físicoanatômicas e de cinética digestiva favorecem ou não o consumo de forragens pelos animais.
Chacon e Stobbs (1976), ao avaliarem o hábito de pastejo de bovinos, no outono e na primavera, verificaram que o tempo de pastejo variou de 9,88 a $10,76 \mathrm{~h}$, a taxa de bocados de 59,4 a 62,4 bocados por minuto, respectivamente, nas duas estações.

Farinatti et al. (2004), ao estudarem o comportamento de pastejo de vacas da raça Holandesa no terço final da lactação, em pastagem natural do Rio Grande do Sul, observaram que o tempo de pastejo variou entre 8,28 e 9,83h, de ruminação entre 5,23 e 9,88h, de ócio entre 3,76 e $6,86 \mathrm{~h}$, e taxa de bocado entre 38 e 43 bocados por minuto, de acordo com a disponibilidade de forragem.

O objetivo deste trabalho foi avaliar o comportamento de pastejo de vacas Holandês $\mathrm{x}$ Zebu, no terço inicial de lactação, em pastagens de Brachiaria brizantha e Brachiaria decumbens.

\section{MATERIAL E MÉTODOS}

O experimento foi realizado na Fazenda Lírio dos Vales, localizada no município de Aragoiania-GO. Foram utilizadas pastagens já formadas de $B$. brizantha e $B$. decumbens. Nas Tab. 1 e 2 encontram-se, respectivamente, a análise de fertilidade e textura do solo e a composição bromatológica dos pastos.

Foram utilizadas 10 vacas Holandês x Zebu no terço inicial de lactação e com média de peso de $510 \mathrm{~kg}$. O sistema de pastejo foi o de lotação contínua, com taxa de lotação variável, com objetivo de manter a altura dos pastos em torno de $30 \mathrm{~cm}$. Para tanto, foram utilizados animais reguladores, tendo em vista que cada pasto compreendeu uma área de 2,0ha.

O período experimental teve duração de 30 dias, sendo 20 para adaptação dos animais ao pasto e 10 para avaliações. Foram feitas três avaliações com duração de $24 \mathrm{~h}$ cada, realizadas a cada cinco dias. As avaliações foram realizadas nos dias 20,25 e 30 de dezembro de 2004. A média das temperaturas foi de $22^{\circ} \mathrm{C}$ durante a noite e de $28^{0} \mathrm{C}$ durante o dia. 
Hábito de pastejo de vacas lactantes...

Tabela 1. Análise do solo da fazenda Lírio dos Vales, Aragoiania, GO, segundo a forrageira

\begin{tabular}{lcc} 
& Pasto de & Pasto de \\
& Brachiaria decumbens & Brachiaria brizantha \\
\hline $\mathrm{pH}\left(\mathrm{CaCl}_{2}\right)$ & 4,60 & 5,80 \\
$\mathrm{Ca}\left(\mathrm{cmol}_{\mathrm{c}} / \mathrm{dm}^{3}\right)$ & 0,90 & 2,50 \\
$\mathrm{Mg}\left(\mathrm{cmol}_{\mathrm{c}} / \mathrm{dm}^{3}\right)$ & 0,50 & 0,96 \\
$\mathrm{Al}\left(\mathrm{cmol}_{\mathrm{c}} / \mathrm{dm}^{3}\right)$ & 0,20 & 0,00 \\
$\mathrm{Zn}\left(\mathrm{cmol}_{\mathrm{c}} / \mathrm{dm}^{3}\right)$ & 0,40 & 0,60 \\
$\mathrm{P}-\mathrm{Mehlich}-1\left(\mathrm{mg} / \mathrm{dm}^{3}\right)$ & 1,50 & 2,20 \\
$\mathrm{~K}-\mathrm{Mehlich}-1\left(\mathrm{mg} / \mathrm{dm}^{3}\right)$ & 41,0 & 35,00 \\
$\mathrm{H}+\mathrm{Al}\left(\mathrm{cmol}_{\mathrm{c}} / \mathrm{dm}^{3}\right)$ & 3,15 & 5,80 \\
$\mathrm{CTC}\left(\mathrm{cmol}_{\mathrm{c}} / \mathrm{dm}^{3}\right)$ & 5,00 & 8,12 \\
$\mathrm{Saturação} \mathrm{por} \mathrm{alumínio}(\%)$ & 11,76 & 0,00 \\
Saturação por bases $(\%)$ & 32,00 & 48,00 \\
Argila $(\%)$ & 37,00 & 39,00 \\
Limo $(\%)$ & 9,00 & 9,00 \\
Areia $(\%)$ & 54,00 & 52,00 \\
Matéria orgânica $(\%)$ & 2,0 & 1,9 \\
\hline
\end{tabular}

Tabela 2. Análise bromatológica (\%) dos pastos da fazenda Lírio dos Vales, Aragoiania, GO

\begin{tabular}{lccc}
\hline Capim & MS & FDN & PB \\
\hline Brachiaria decumbens & 33,80 & 67,80 & 6,73 \\
Brachiaria brizantha & 35,06 & 63,02 & 8,36 \\
\hline
\end{tabular}

$\mathrm{MS}=$ matéria seca; FDN=fibra em detergente neutro; $\mathrm{PB}=$ proteína bruta.

O delineamento experimental foi o inteiramente ao acaso, sendo os tratamentos as pastagens de Brachiaria brizantha e Brachiaria decumbens, com cinco repetições (cinco animais por tratamento). As variáveis analisadas foram: tempo total de pastejo (TTP), tempo de pastejo diurno (TPD), tempo de pastejo noturno (TPN), tempo total de ruminação (TTR), tempo de ruminação diurno (TRD), tempo de ruminação noturno (TRN), tempo total de ócio (TTO), tempo de ócio diurno (TOD), tempo de ócio noturno (TON), quantidade de bocados por minuto (QBM) e total de bocados (TB).

Os tempos de pastejo, ruminação e ócio foram obtidos por meio de observações visuais dos animais a cada 10 minutos, sendo o tempo total o somatório do total de vezes nas quais os animais foram observados em determinado estado. Considerou-se como período diurno entre 7 e $18 \mathrm{~h}$ e noturno entre 19 e $6 \mathrm{~h} 50 \mathrm{~min}$.

A taxa de bocados (QBM) foi obtida por meio da contagem direta do total de bocados observados no período de um minuto, sendo a resultante da média de observações a cada meia hora, quando os animais estavam pastejando. A quantidade total de bocados (QTB) foi calculada pelo produto entre a taxa de bocados e o tempo de pastejo, em minutos.

Os dados referentes aos tempos de pastejo, ócio e ruminação e a taxa e total de bocados observados nos dois pastos foram submetidos à análise de variância com $\mathrm{P}<0,05$. As análises foram realizadas utilizando-se o pacote estatístico SAEG (Sistema..., 1999).

\section{RESULTADOS E DISCUSSÃO}

As Fig. 1 e 2 mostram as variações diárias no comportamento de pastejo das vacas nos dois pastos. O pastejo foi intenso durante todo o período da manhã, semelhantes às observações de Van Soest (1994). A partir das 16h, os animais reduziram a atividade de pastejo e intensificaram as atividades de ruminação. A permanência dos animais em ócio foi observada praticamente no final da madrugada até o amanhecer. Os resultados indicam que a ordenha pouco antes do amanhecer é satisfatória, tendo em vista que a intensificação do pastejo vai ocorrer após o amanhecer, quando os animais estariam deixando as atividades de ordenha. 


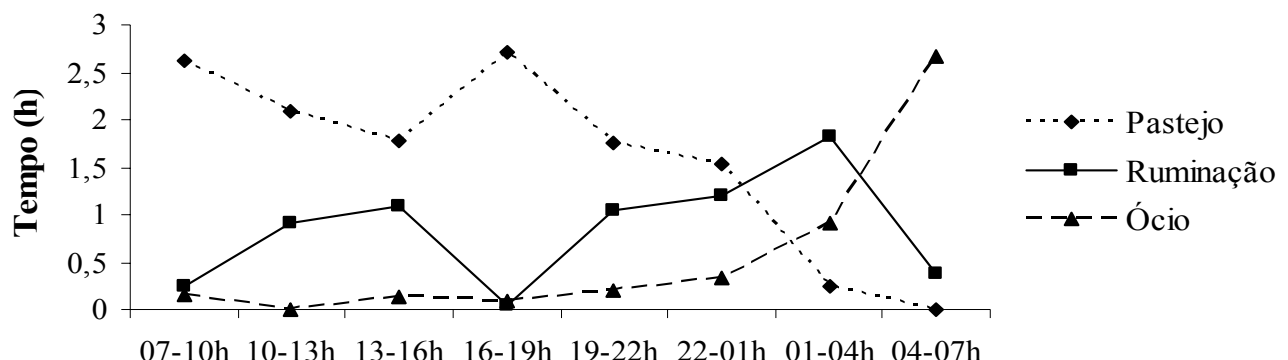

\section{Horário do dia}

Figura 1. Variação no comportamento diário de pastejo, ruminação e ócio das vacas no pasto de Brachiaria decumbens.

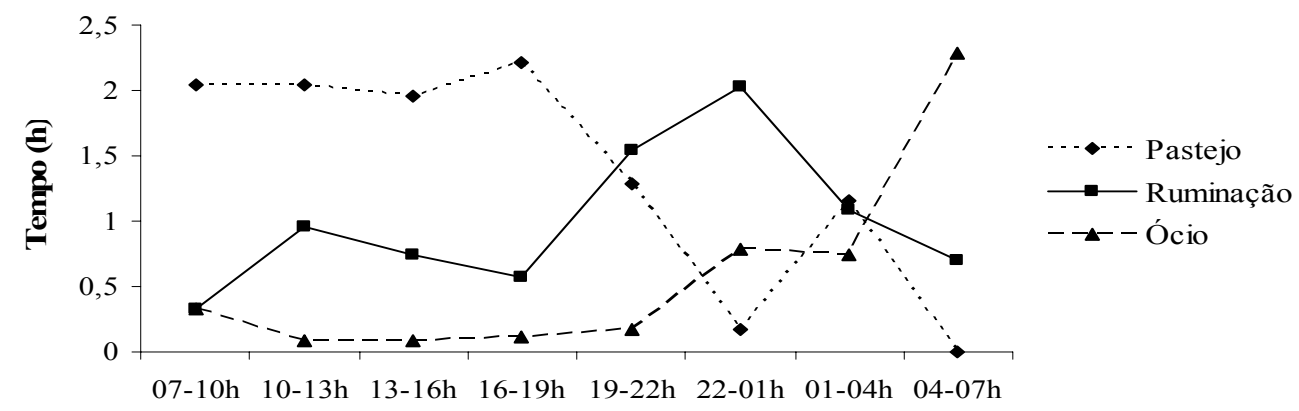

Horário do dia

Figura 2. Variação no comportamento diário de pastejo, ruminação e ócio das vacas no pasto de Brachiaria brizantha.

Quanto ao tempo de pastejo (Tab. 3), não se observou diferença significativa entre os dois pastos no período diurno, entretanto o mesmo não ocorreu durante a noite, desse modo, para o período total, o tempo de pastejo foi maior no pasto de $B$. decumbens. Isso, provavelmente, deve-se ao seu mais baixo valor nutritivo, com menor quantidade de $\mathrm{PB}$ e maior conteúdo de FDN (Tab. 2), o que, segundo Olivo et al. (2004), força o animal a exercer um pastejo mais seletivo. A $B$. brizantha é considerada uma gramínea de melhor qualidade quando comparada com a $B$. decumbens, além de ser uma espécie de porte mais alto. Como os dois pastos foram manejados na mesma altura, a arquitetura da $B$. decumbens pode ter levado ao pastejo mais seletivo. Segundo Sarmento (2003), a estrutura do dossel forrageiro modifica o hábito de pastejo dos animais, de modo que as diferenças entre duas espécies com porte diferente sejam esperadas.
Lima et al. (2000), ao avaliarem o comportamento de pastejo de vacas mestiças Gir $\mathrm{x}$ Holandês, em pastagem de capim-elefante (Pennisetum purpureum) e capim-tanzânia (Panicum maximum), observaram tempo de pastejo de 7,67 e 7,23h, respectivamente, valores menores que os observados no presente experimento, para as duas pastagens estudadas. Esses autores não avaliaram a freqüência de bocados, todavia a diferença no tempo de pastejo pode ser atribuída à menor freqüência de bocados, o que é esperado em pastagens de porte mais elevado. Werneck et al. (2000), ao estudarem o comportamento de vacas mestiças Gir x Holandês em pastagem de capim-elefante, observaram tempo de pastejo de $8,4 \mathrm{~h}$, um pouco maior que o obtido por trabalho de Lima et al. (2004), porém menor que o do presente trabalho. 
Farinatti et al. (2004), ao avaliarem o comportamento de pastejo de vacas da raça Holandesa no terço final da lactação em pastagem natural, no Rio Grande do Sul, observaram tempo de pastejo variando de 8,28 a $9,83 \mathrm{~h}$, valores próximos aos observados neste trabalho. Resultados semelhantes foram observados por Chacon e Stobbs (1976).

Tabela 3. Tempo de pastejo (horas) das vacas segundo a pastagem e a hora do dia, e respectivo coeficiente de variação

\begin{tabular}{lccc} 
Capim & TPD & TPN & TTP \\
\hline Brachiaria brizantha & $8,24 \mathrm{a}$ & $2,58 \mathrm{~b}$ & $10,82 \mathrm{~b}$ \\
Brachiaria decumbens & $9,24 \mathrm{a}$ & $3,95 \mathrm{a}$ & $12,86 \mathrm{a}$ \\
$\mathrm{CV}(\%)$ & 7,62 & 9,32 & 6,63
\end{tabular}

Médias seguidas por letras distintas na coluna diferem entre si $(\mathrm{P}<0,05)$.

$\mathrm{TPD}=$ tempo de pastejo diurno; $\mathrm{TPN}=$ tempo de pastejo noturno; TTP=tempo total de pastejo.

Na Tab. 4 observam-se os tempos de ruminação. As diferenças ocorreram somente no período noturno e, conseqüentemente, no total. Diferentemente do observado para o tempo de pastejo, os tempos de ruminação nos período noturno e total foram menores para a $B$. decumbens. Essa diferença também se deve ao comportamento mais seletivo dos animais nessa pastagem, ao gastarem mais tempo selecionando a dieta. Provavelmente, ingeriram dieta com maior quantidade de folhas, de maneira que necessitaram de menor tempo em ruminação.

Farinatti et al. (2004) observaram que vacas da raça Holandesa ruminaram 5,2h por dia em pastagem de capim coast-cross, enquanto Pires (1997), ao avaliar o comportamento de vacas lactantes com esse mesmo capim, verificou tempo de ruminação de $3,36 \mathrm{~h}$, resultado menor que os observados para os dois pastos estudados neste experimento. Voltolini et al. (2003) observaram que vacas em lactação em pastejo de capim-elefante gastaram 8,06 horas, resultado mais alta que o observado para a $B$. decumbens, e muito próximo aos verificados para $B$. brizantha.

Lima (2002), ao estudar o hábito de pastejo de vacas mestiças em pastagens de capim-elefante e capim-tanzânia, observou tempo de ruminação de $5,7 \mathrm{~h}$, resultado menor que os observados no presente trabalho. Assim, parece que há uma adequação entre o tempo de pastejo e de ruminação de acordo com a forragem pastejada.

Tabela 4. Tempo de ruminação (horas) das vacas segundo a pastagem e a hora do dia e respectivo coeficiente de variação

\begin{tabular}{lccc}
\hline Capim & TRD & TRN & TTR \\
\hline Brachiaria brizantha & $2,58 \mathrm{a}$ & $5,37 \mathrm{a}$ & $7,95 \mathrm{a}$ \\
Brachiaria decumbens & $2,29 \mathrm{a}$ & $4,16 \mathrm{~b}$ & $6,45 \mathrm{~b}$ \\
CV $(\%)$ & 17,66 & 8,43 & 6,43 \\
\hline
\end{tabular}

Médias seguidas de letras distintas na coluna diferem entre si $(\mathrm{P}<0,05)$.

$\mathrm{TRD}=$ tempo de ruminação diurno; TRN=tempo de ruminação noturno; TTR=tempo total de ruminação.

Na Tab. 5 observam-se os tempos de ócio. Não foram observadas diferenças entre os dois pastos, o que significa que houve adequação dos animais com relação ao pastejo e à ruminação, de maneira que os tempos de ócio fossem muito próximos para os dois pastos. A maioria do tempo de ócio foi observada durante a noite, com valores equivalentes a 87 e $91 \%$ do total para os pastos de $B$. brizantha e $B$. decumbens, respectivamente.
Martinez et al. (2004), ao estudarem o comportamento ingestivo de vacas da raça Holandesa pastejando capim-elefante durante a estação chuvosa, verificaram que os animais permaneceram $7,2 \mathrm{~h}$ em pastejo, muito superior ao observado no presente experimento. Valores mais altos também foram observados por Gibb et al. (1999), com tempo de 6,66h para vacas lactantes. No trabalho de Farinatti et al. (2004), os valores de ócio variaram entre 3,76 e $6,86 \mathrm{~h}$. 
Tabela 5. Tempo de ócio (horas) das vacas segundo a pastagem e a hora do dia e respectivo coeficiente de variação

\begin{tabular}{lccc} 
& \multicolumn{3}{c}{ Tempo de ócio (horas) } \\
\cline { 2 - 4 } Capim & TOD & TON & TTO \\
\hline Brachiaria brizantha & $0,58 \mathrm{a}$ & $3,99 \mathrm{a}$ & $4,57 \mathrm{a}$ \\
Brachiaria decumbens & $0,37 \mathrm{a}$ & $4,12 \mathrm{a}$ & $4,49 \mathrm{a}$ \\
CV $(\%)$ & 60,59 & 10,14 & 12,63 \\
\hline
\end{tabular}

Médias seguidas por letras distintas na coluna diferem entre si $(\mathrm{P}<0,05)$.

TOD=tempo de ócio diurno; $\mathrm{TON}=$ tempo de ócio noturno; $\mathrm{TTO}=$ tempo total de ócio.

Com relação à taxa e ao total de bocados, na Tab. 6 mostra-se que a QBM foi maior para o pasto de $B$. brizantha, o que pode ser considerado como um efeito compensador do menor tempo de pastejo. Conseqüentemente, QTB foi o mesmo para os dois pastos, demonstrando claramente que os animais aumentaram o tempo de pastejo para compensar o comportamento seletivo praticado na Brachiaria decumbens.
Martinez et al. (2004) encontraram valores de 35,6 bocados por minuto para vacas da raça Holandesa em pastagem de capim-elefante, valor inferior aos observados neste experimento, o que pode ser atribuído a diferenças na estrutura dos pastos. Farinatti et al. (2004) encontraram valores de 37 a 39 bocados por minuto, com um total de bocados entre 17.340 e 18.266 por dia, valores inferiores aos observados neste experimento.

Tabela 6. Quantidade de bocados por minuto e total de bocados de vacas segundo a pastagem e respectivo coeficiente de variação

\begin{tabular}{lcc}
\hline Capim & QTB & QBM \\
\hline Brachiaria brizantha & $27369 \mathrm{a}$ & $42,12 \mathrm{a}$ \\
Brachiaria decumbens & $28144 \mathrm{a}$ & $37,30 \mathrm{~b}$ \\
$\mathrm{CV}(\%)$ & 8,46 & 2,37
\end{tabular}

Médias seguidas por letras distintas na coluna diferem ente si $(\mathrm{P}<0,05)$.

$\mathrm{QTB}=$ quantidade total de bocados; $\mathrm{QNM}=$ quantidade de bocados por minuto.

\section{CONCLUSÕES}

As vacas modificam o comportamento de pastejo, como forma de compensar diferenças na estrutura e valor nutritivo dos pastos de $B$. brizantha e $B$. decumbens, aumentando o tempo de pastejo e a quantidade total de bocados no pasto de $B$. decumbens, para compensar o comportamento mais seletivo neste pasto.

\section{REFERÊNCIAS BIBLIOGRÁFICAS}

CARVALHO, P.C.F.; POLI, C.H.A.C.; NABINGER, C. et al. Comportamento ingestivo de bovinos em pastejo e sua relação com a estrutura da pastagem. In: PECUÁRIA 2000: A PECUÁRIA DE CORTE NO III MILÊNIO, 2000, Pirassununga, SP. Anais... Pirassununga: FZEA/USP, 2000. CDROM.
CHACON, E.; STOBBS, T. H. Influence of progressive defoliation of a grass sward on the eating behaviour of cattle. Austr. J. Agric. Res. v.27, p.709-727, 1976.

FARINATTI, L. H.; POLI, C. H. A. C.; MONKS, P. L. et al. Comportamento ingestivo de vacas holandesas em sistemas de produção de leite a pasto na região da Campanha do Rio Grande do Sul. In: REUNIÃO ANUAL DA SOCIEDADE BRASILEIRA DE ZOOTECNIA, 2004, Campo Grande. Anais... Campo Grande: SBZ, 2004. CDROM.

GIBB, M.J.; HUCKLE, C.A.; NUTHALL, R. et al. The effect of physiological state lactating or dry and sward surface height on grazing behaviour and intake by dairy cows. Appl. Anim. Behav. Sci., v.63, p.269-287, 1999.

LIMA, M.L.P. Produção de leite de vacas mestiças em pastagem de capim elefante $c v$. Guaçu (Pennisetum purpureum Schum. Cv. 
Guaçu) e capim tanzânia (Panicum maximum Jacq. cv. Tanzânia). 2002. 102f. Dissertação (Mestrado em Zootecnia) - Universidade Estadual Paulista, Jaboticabal, SP.

LIMA, N. C.; SIMILI, F.F.; LIMA, M.L.P. et al. Tempo de pastejo de vacas mestiças em sistema rotacionado de capim-tanzânia (Panicum maximum) e capim-elefante (Pennisetum purpureum). In: REUNIÃO ANUAL DA SOCIEDADE BRASILEIRA DE ZOOTECNIA, 2000, Viçosa. Anais.... Viçosa: SBZ, 2000. CDROM.

MARTINEZ, J.C.; SANTOS, F.A.P.; VOLTOLINI, T.V. et al. Comportamento ingestivo de vacas leiteiras pastejando capimelefante durante a estação chuvosa. In: GRASSLAND ECOPHYSIOLOGY AND GRAZING ECOLOGY, 2004, Curitiba, Proceedings... Curitiba: UFP, 2004. CDROM.

MINSON, D.J.; WILSON, J.R. Prediction of intake as an element of forage qualitty. In: NATIONAL CONFERENCE ON FORAGE QUALITTY: FORAGE QUALITY, EVALUATION, AND UTILIZATION, 1994, Madson. Proceedings... Madson: ASA, 1994. p.533-563.

OLIVO, J. C.; CHARÃO, P. S.; SOBCZAK, M. S. et al. Comportamento de vacas da raça Holandesa em pastagem manejada sob princípios agroecológicos. In: REUNIÃ̃ ANUAL DA SOCIEDADE BRASILEIRA DE ZOOTECNIA, 2004, Campo Grande. Anais... Campo Grande: SBZ, 2004. CDROM.

PIRES, M.F.A. Comportamento, parâmetros fisiológicos e reprodutivos de fêmeas da raça holandesa confinadas em free-stall, durante o verão e inverno. 1997. 151f. Tese (Doutorado em Zootecnia) - Escola de Veterinária, Universidade Federal de Minas Gerais, Belo Horizonte, MG.

RIBEIRO, H.M.N.; ALMEIDA, E.X.; HARTHMANN, O.E.L. et al. Tempo e ciclos diários de pastejo de bovinos submetidos a diferentes ofertas de forragem de capim-elefante anão cv. Mott. In: REUNIÃO ANUAL DA SOCIEDADE BRASILEIRA DE ZOOTECNIA, 1997, Juiz de Fora. Anais... Juiz de Fora: SBZ, 1997. p.274-275.

SARMENTO, D.O.L. Comportamento ingestivo de bovinos em pastos de capim-Marandu submetidos a regimes de lotação contínua. 2003. 76f. Dissertação (Mestrado em Zootecnia) Escola Superior de Agricultura Luiz de Queiroz, Universidade de São Paulo, Piracicaba, SP.

SISTEMA de Análises Estatísticas e Genéticas SAEG. Viçosa: UFV, 1999. Manual do usuário, 138p. (versão 8.0).

TREVISAN, N.B.; QUADROS, F.L.F.; CORADINI, F.S. et al. Comportamento ingestivo de novilhos de corte em pastagem de aveia preta e azevém com níveis distintos de folhas verdes. Cienc. Rural, v.34, p.1543-1548, 2004.

VAN SOEST, P. J. Nutritional ecology of the ruminant. 2.ed. Cornell University, 1994. 476p.

VOLTOLINI, T.V.; SANTOS, F.A.P.; MARTINEZ, J.C. Consumo de matéria seca e comportamento ingestivo de vacas em lactação mantidas em pastagem de capim elefante, recebendo suplementação com concentrado com diferentes teores de proteína bruta. In: REUNIÃO ANUAL DA SOCIEDADE BRASILEIRA DE ZOOTECNIA, 2003, Santa Maria. Anais... Santa Maria: SBZ, 2003. CDROM.

WERNECK, C.L.; WERNEQUE, R.S.; PIRES, M.F.A. Comportamento alimentar das raças mestiças (Holandês x Zebu), em pastagem de capim-elefante (Pennisetum purpureum). In: ENCONTRO ANUAL DE ETOLOGIA, 2000, Florianópolis. Anais... Florianópolis: SBET, 2000. p.74 